\title{
When is an Interacting Particle System Ergodic?
}

\author{
Christian Maes ${ }^{1}$ and Senya B. Shlosman ${ }^{2}$ \\ Institute for Problems of Information Transmission, Moscow \\ Received December 16, 1991; in revised form July 28, 1992
}

\begin{abstract}
We consider stochastic flip dynamics for an infinite number of Ising spins on the lattice $\mathbb{Z}^{d}$. We find a sequence of constructive criteria for the system to be exponentially ergodic. The main idea is to approximate the continuous time process with discrete time processes (its Euler polygon) and to use an improved version of previous results [MS] about constructive ergodicity of discrete time processes.
\end{abstract}

\section{Introduction}

Ever since the appearance in probability theory of random processes defined through the interaction of infinite interacting particle systems ( $[S, V, D])$, such general questions as in the title have been investigated. The problem is to consider a broad family of systems or automata containing a large number of interacting components and to give criteria under which their behavior is essentially the same as for non-interacting processes. As observed already by Tolstoi (and quoted in $[\mathrm{DS}])$, there are general reasons for "happiness" in a family. Here, this is called ergodic behavior and, depending on the context, also stands for unreliability, memory loss, convergence to the unique invariant measure, high noise regime, absence of phase transition and more of that. Its counterpart in equilibrium statistical mechanics is the so-called high temperature regime, for which the technique of cluster expansions and Dobrushin-Shlosman analysis have given complete characterization.

As is often the case, giving general answers and complete descriptions is often limited to cases of less interest. Critical behavior, catastrophes and bifurcations, coexistence of phases and all that, are in some sense perpendicular to the ergodic

\footnotetext{
1 Aangesteld Navorser N.F.W.O., Instituut voor Theoretische Fysica, K.U. Leuven, Celestijnenlaan 200D, 3001 Leuven, Belgium

${ }^{2}$ Institute for the Problems of Information Transmission, Moscow, and Department of Mathematics, UCI, Irvine, CA 92717, USA
} 
regime. It becomes however more interesting if the criteria for ergodicity can be made optimal and ideally, touch the regime where critical phenomena appear. Such analysis was carried out for other systems such as in percolation theory [ACCFR] or for ferromagnetic lattice gas models [A]. The idea is reminiscent of the renormalization group: looking at big enough (but finite) parts one discovers via a rescaling argument properties of the infinite system. The reasoning is most often of the form: if something happens in a fixed large volume, then we know how the infinite system behaves and vice versa.

In the present paper we take up these old questions for the so-called spinflip processes (the class of Interacting Particle Systems introduced in Sect. 2). These are continuous time processes, where each of the infinitely many spins influences the behavior of any other spin after any amount of time, however small. Still we are able to construct a sequence of finite systems evolving in discrete time, such that the proper behavior of any of these in a finite time interval (which can be effectively verified), ensures the ergodic behavior of the initial IPS.

The opposite is also true, at least for attractive systems: if the initial process is exponentially ergodic, then some systems from our sequence do behave properly. Hence our ergodicity criterium is genuinely constructive: in case one is interested in checking the ergodicity of a process, which, in fact, is exponentially ergodic, one will do that rigorously by working hard and checking more of our conditions. If one is not too lazy, one will succeed.

In implementing our program we connect two recent ideas, both of which we present in an improved version. First, there is the technique of [MS] where the same question was studied for discrete time processes, the so-called Probabilistic Cellular Automata (PCA). Secondly, we use the idea of [St] of approximating continuous time processes by PCA. Summarized, our main idea to prove exponential ergodicity for continuous time processes is to find PCA which are

i) exponentially ergodic,

ii) approximate the initial IPS.

Section 2 has the necessary definitions and notations. We there also remind the reader of the use of coupling which will play a major role in the following sections. Our main results (Theorem A and B) are presented in Sect. 3. To arrive at them we start in Sect. 4 revisiting the question of exponential ergodicity for PCA which was solved in [MS]. We give a very simple (compared to $[\mathrm{MS}]$ ) set of sufficient conditions for the exponential ergodicity of PCA, which gives most of what one can hope for in the discrete time case. They are also necessary conditions for attractive systems. Examples show how new and better rigorous bounds on the critical value of certain interaction parameters can be obtained. The non-locality (in a certain sense) of the continuous time process seemingly is an obstacle to give any constructive criteria for its ergodicity. The extent to which it is non-local is estimated in Sect. 5. Then enters the idea of [St] to approximate IPS via discrete time systems. A simplified approach is contained in Sect. 6 and we get better bounds than in [St]. Each of Sects. 4, 5 or 6 is more or less self-contained. In Sect. 7, we conclude the proof of our main results. 


\section{Definitions and Notations}

a) The state space. An infinite spin configuration $\eta$ on the $d$-dimensional lattice $\mathbb{Z}^{d}$ is an element of the state space $\Omega=\{-1,1\} \mathbb{Z}^{d}$. The spin value at a site $i \in \mathbb{Z}^{d}$ in configuration $\eta \in \Omega$ is denoted by $\eta(i)= \pm 1$. with

The configuration obtained from $\eta$ by flipping the spin at site $j \in \mathbb{Z}^{d}$ is $\eta^{j} \in \Omega$

$$
\eta^{j}(i)=\left\{\begin{aligned}
-\eta(i) & \text { if } i=j, \\
\eta(i) & \text { if } i \neq j
\end{aligned}\right.
$$

The translation $\tau_{i}(\eta), i \in \mathbb{Z}^{d}$, of $\eta \in \Omega$ is a new configuration for which for $j \in \mathbb{Z}^{d}$,

$$
\tau_{i}(\eta)(j)=\eta(i+j) .
$$

Equipping $\Omega$ with the usual product topology, we let $C(\Omega)$ denote the Banach space of all real valued continuous functions $f$ with norm

$$
\|f\|=\sup _{\eta \in \Omega}|f(\eta)| \text {. }
$$

Similar notations will be used when dealing with $k$-tuples $\left(\eta_{1}, \ldots, \eta_{k}\right) \in \Omega^{k}$, $k=1,2, \ldots$ of configurations.

The dependence of a function $f \in C(\Omega)$ on the spin value at $i \in \mathbb{Z}^{d}$ is measured by

$$
\delta_{i} f=\sup _{\eta \in \Omega}\left|f\left(\eta^{i}\right)-f(\eta)\right|
$$

while its total oscillation is

$$
\|f \mid\|=\sum_{i} \delta_{i} f
$$

The set

$$
D(\Omega)=\{f \in C(\Omega): \quad\|f\|<\infty\}
$$

certainly contains all local functions, i.e. functions $f \in C(\Omega)$ such that $f(\eta)=f\left(\eta^{i}\right)$, $\forall \eta \in \Omega$, for all but a finite number of $i \in \mathbb{Z}^{d}$.

b) The discrete time process. Let a function $p_{o}: \Omega \rightarrow[0,1]$ be specified with the property that it only depends on the configuration $\eta \in \Omega$ in a finite neighborhood $U \subset \mathbb{Z}^{d}$ of the origin, i.e.,

$$
p_{o}\left(\eta^{j}\right)=p_{o}(\eta) \quad \text { whenever } j \notin U .
$$

The range of interaction is the smallest number $r<\infty$ for which

$$
U \subset([-r, r] \cap \mathbb{Z})^{d} .
$$

The translates $p_{i}: \Omega \rightarrow[0,1], i \in \mathbb{Z}^{d}$, are defined via

$$
p_{i}(\eta)=p_{o}\left(\tau_{i}(\eta)\right)
$$

only depending on the spin values $\eta(j), j \in U+i$.

Let $p(d \sigma \mid \eta)$ be the product probability measure on $\Omega$ with probabilities $\operatorname{Prob}[\sigma(i)=1 \mid \eta]=p_{i}(\eta)$, i.e. for every finite set $A \subset \mathbb{Z}^{d}$,

$$
\int \prod_{i \in A} \sigma(i) p(d \sigma \mid \eta)=\prod_{i \in A}\left(2 p_{i}(\eta)-1\right) .
$$


The Probabilistic Cellular Automation (PCA) determined by $p_{o}$ is the discrete time Markov process $\left\{\sigma_{n}\right\}_{n>0}$ with state space $\Omega$ and transition probabilities $p(d \sigma \mid \eta)$. The transition operator $P$ is defined on all $f \in C(\Omega)$ as

$$
P f(\eta)=\int f(\sigma) p(d \sigma \mid \eta) .
$$

A probability measure $v$ on $\Omega$ evolves according to

$$
v_{n}=v_{n-1} P=v P^{n}, \quad n \geqq 1
$$

with $v_{0}=v$ and for any probability measure $\mu$

$$
\int f(\sigma) \mu P(d \sigma)=\int P f(\sigma) \mu(d \sigma) .
$$

We say that the PCA is attractive if $p_{i}(\eta)$ is a non-decreasing function of $\eta$, i.e. $p_{i}(\eta) \geqq p_{i}\left(\eta^{j}\right)$ if $\eta(j)=1$.

A stationary or invariant measure $v$ solves

$$
v P=v,
$$

while we say that the PCA is ergodic whenever, for all initial measures $\mu$, there is convergence to the unique invariant measure:

$$
\mu_{n} \rightarrow v, \quad \text { weakly }
$$

as $n \uparrow \infty$.

It is convenient to introduce also the space-time lattice $\mathbb{Z}^{d+1}$ of points $x=(n, i)$, $n \in \mathbb{Z}, \quad i \in \mathbb{Z}^{d}$, and the space $\Omega^{\infty}=\{-1,+1\}^{\mathbb{Z}^{d+1}}$ of space-time configurations $\sigma=\left\{\sigma_{x}, x \in \mathbb{Z}^{d+1}\right\}, \sigma_{x}= \pm 1$. The restriction of such a $\sigma \in \Omega^{\infty}$ to a volume $V \subset \mathbb{Z}^{d+1}$ is $\sigma_{V}=\left\{\sigma_{x}, x \in V\right\}$, and, as a special case we view $\sigma_{n}=\left\{\sigma_{x}=\sigma_{n}(i)\right.$, $\left.x=(n, i), i \in \mathbb{Z}^{d}\right\} \in \Omega$ as its $n^{\text {th }}$ time layer restriction.

c) The continuous time process. The continuous time analogs of the PCA introduced above are called Interacting Particle Systems (IPS) and are described by giving for each $i \in \mathbb{Z}^{d}$ a local non-negative and bounded function $c(i, \eta), \eta \in \Omega$, which is to be interpreted as the probability per unit time of flipping the spin at site $i$ if the system is in state $\eta$. That is,

$$
\operatorname{Prob}\left[\eta_{t}(i) \neq \eta(i) \mid \eta_{0}=\eta\right]=c(i, \eta) t+o(t) .
$$

In analogy with the PCA construction we require that

$$
\begin{aligned}
c(i, \eta) & =c\left(o, \tau_{i}(\eta)\right) \geqq 0, \\
c\left(i, \eta^{j}\right) & =c(i, \eta) \quad \text { whenever } j \notin U+i,
\end{aligned}
$$

and let

$$
\begin{aligned}
B & =\sup _{\eta \in \Omega} c(o, \eta)<\infty \\
M & =\sum_{j \neq o} \sup _{\eta}\left|c\left(o, \eta^{j}\right)-c(o, \eta)\right| \\
\varepsilon & =\inf _{\eta}\left[c(o, \eta)+c\left(o, \eta^{o}\right)\right] .
\end{aligned}
$$


On a local function $f(\eta), \eta \in \Omega$, the generator $L$ of this continuous time Markov spinflip process is acting as

$$
L f(\eta)=\sum_{i \in \mathbb{Z}^{d}} c(i, \eta)\left[f\left(\eta^{i}\right)-f(\eta)\right] .
$$

The corresponding Markov semi-group will be denoted by $S(t), t \geqq 0$. See [L] for the details of the construction.

We say that the IPS is attractive if $c(o, \eta) \geqq c(o, \sigma)$ if $\eta(o)=\sigma(o)=-1$ and $c(o, \eta) \leqq c(o, \sigma)$ if $\eta(o)=\sigma(o)=1$ for any two configurations $\eta, \sigma \in \Omega$ for which $\eta(i) \geqq \sigma(i), \forall i \in \mathbb{Z}^{d}$.

As before, a probability measure $v$ on $\Omega$ is stationary or invariant if

$$
v S(t)=v,
$$

and the process is said to be ergodic whenever for all initial measures $\mu, \mu S(t) \rightarrow v$, weakly as $t \uparrow \infty$.

d) Coupling of PCA and IPS. Our main results are formulated in terms of couplings of certain measures. We start by reminding the reader the definition of the most simple one for PCA: the discrete time Vasserstein coupling.

Fix a function $a_{o}$ on $\Omega \times \Omega$ :

$$
a_{o}(\eta, \bar{\eta})=\min \left\{p_{o}(\eta), p_{o}(\bar{\eta})\right\}, \quad \eta, \bar{\eta} \in \Omega .
$$

A probability measure $q_{o}(\cdot \mid \eta, \bar{\eta})$ on $\{-1,1\} \times\{-1,1\}$ is defined by putting (in short-hand notation)

$$
\begin{aligned}
& q_{o}(+,+\mid \eta, \bar{\eta})=a_{o}(\eta, \bar{\eta}) \\
& q_{o}(+,-\mid \eta, \bar{\eta})=p_{o}(\eta)-a_{o}(\eta, \bar{\eta}) \\
& q_{o}(-,+\mid \eta, \bar{\eta})=p_{o}(\bar{\eta})-a_{o}(\eta, \bar{\eta}) \\
& q_{o}(-,-\mid \eta, \bar{\eta})=1-p_{o}(\eta)-p_{o}(\bar{\eta})+a_{o}(\eta, \bar{\eta}) .
\end{aligned}
$$

By translation this also defines $q_{i}(\cdot \mid \eta, \bar{\eta})$ for every $i \in \mathbb{Z}^{d}$.

Let $q(d \sigma, d \bar{\sigma} \mid \eta, \bar{\eta})$ denote the product measure on $(\{-1,1\} \times\{-1,1\})^{\mathbb{Z}^{d}}$ $\cong \Omega \times \Omega$ with marginals $q_{i}(\cdot \mid \eta, \bar{\eta}), i \in \mathbb{Z}^{d}$. Clearly, $q(d \sigma, d \bar{\sigma} \mid \eta, \bar{\eta})$ is a coupling measure for $p(d \sigma \mid \eta)$ and $p(d \sigma \mid \bar{\eta})$. The associated coupling PCA on $\Omega \times \Omega$ is defined via its transition operator $Q$, on $f \in C(\Omega \times \Omega)$,

$$
Q f(\eta, \bar{\eta})=\int f(\sigma, \bar{\sigma}) q(d \sigma, d \bar{\sigma} \mid \eta, \bar{\eta}) .
$$

Again, if $f(\sigma, \bar{\sigma})=g(\sigma)$, then $\quad Q f(\eta, \bar{\eta})=P g(\eta) ; \quad$ if $f(\sigma, \bar{\sigma})=g(\bar{\sigma})$, then $Q f(\eta, \bar{\eta})=g(\bar{\eta})$.

Remark. Other couplings can be constructed by choosing in (2.22) $0 \leqq a_{o} \leqq \min \left\{p_{o}(\eta), p_{o}(\bar{\eta})\right\}$. While we will restrict ourselves in the following to the Vasserstein coupling, the results of Sect. 4 can be formulated using any one of those other couplings.

Consider the strip $S_{N}=\mathbb{Z}^{d} \times\{0,1, \ldots, N\}$ of time length $N \geqq 1$. For all initial data $\eta \in \Omega$, let $\mu_{N}(d \sigma \mid \eta)$ be the probability measure on $S_{N}$ obtained from the PCA 
(2.7)-(2.11). That is, for a function $f \in C\left(\Omega^{N}\right)$,

$$
\begin{aligned}
& \int f\left(\sigma_{1}, \ldots, \sigma_{N}\right) \mu_{N}(d \sigma \mid \eta) \\
& \quad=\int_{\Omega} \cdots \int_{\Omega} \int_{\Omega} f\left(\sigma_{1}, \ldots, \sigma_{N}\right) p\left(d \sigma_{N} \mid \sigma_{N-1}\right) p\left(d \sigma_{N-1} \mid \sigma_{N-2}\right) \ldots p\left(d \sigma_{1} \mid \eta\right) .
\end{aligned}
$$

Consider now the coupling PCA defined above. The analog of (2.24) defines probability measures $\rho_{N}(d \sigma, d \bar{\sigma} \mid \eta, \bar{\eta})$ on the same strip $S_{N}$.

By construction $\rho_{N}(d \sigma, d \bar{\sigma} \mid \eta, \bar{\eta})$ is a coupling of $\mu_{N}(d \sigma \mid \eta)$ and $\mu_{N}(d \sigma \mid \bar{\eta})$. How this coupling behaves for the spin at site $i \in \mathbb{Z}^{d}$ at time $N$ is obtained by letting $g_{i} \in C\left(\Omega^{N} \times \Omega^{N}\right)$ be given as

$$
\begin{aligned}
g_{i}(\sigma, \bar{\sigma}) & =1 & & \text { if } \sigma_{N}(i) \neq \bar{\sigma}_{N}(i) \\
& =0 & & \text { otherwise },
\end{aligned}
$$

and putting

$$
Q^{\eta, \bar{\eta}}\left[\sigma_{N}(i) \neq \bar{\sigma}_{N}(i)\right]=\int g_{i}(\sigma, \bar{\sigma}) \rho_{N}(d \sigma, d \bar{\sigma} \mid \eta, \bar{\eta}) .
$$

This notation will be frequently used in the rest of the paper also for other couplings.

In the continuous time case, for the IPS defined in (2.16)-(2.19), we refer to III.1 in $[\mathrm{L}]$ for a definition of the so-called basic coupling or Vasserstein coupling. It is a new process $T^{\eta \cdot \sigma}$ on the product space $\Omega \times \Omega$ with marginals the IPS started from configuration $\eta$ and the same IPS but started from configuration $\sigma$. An important feature is that for attractive IPS

$$
\begin{aligned}
T^{\eta, \eta^{j}}\left[\eta_{t}(o) \neq\left(\eta^{j}\right)_{t}(o)\right] & =\left|S(t)(\eta)-S(t)\left(\eta^{j}\right)\right| \\
& \leqq S(t)(+)-S(t)(-),
\end{aligned}
$$

where for the IPS $S(t)(\sigma)=\operatorname{Prob}\left[\sigma_{t}=1 \mid \sigma_{0}=\sigma\right]$ and the arguments \pm refer to the all plus, respectively, all minus configuration. Similar formulae hold for attractive PCA as well (replacing in (2.27) $T$ by $Q$ and $S(t)$ by $P^{n}$ ).

\section{Main Results}

Let an IPS be given as defined in (2.16)-(2.19). For a given $0<\delta$, define a $\delta$ approximating PCA ( $\delta$ PCA) by putting for $(2.9)$

$$
\begin{aligned}
p_{i}^{(\delta)}(\eta) & =\frac{1}{2}(1-\exp [-2 \delta c(i, \eta)]) & & \text { if } \eta(i)=-1 \\
& =1-\frac{1}{2}(1-\exp [-2 \delta c(i, \eta)]) & & \text { if } \eta(i)=1
\end{aligned}
$$

The super- (or sometimes sub-) script $\delta$ is used also for other quantities to identify this $\delta$ PCA.

Fix $N=1,2, \ldots$. Consider the function

$$
z(N, \delta)=\frac{B}{M}\left(e^{M N \delta}-1\right)-\frac{1-e^{-2 B \delta}}{2} \frac{e^{M N \delta}-1}{e^{M \delta}-1} .
$$


As will be shown in Sect. 6, (3.2) is an upper bound on the closeness between the IPS after continuous time $N \delta$ and its $\delta$ PCA after $N$ discrete time steps, provided they both start from the same configuration.

We further define for integer $n \geqq 1$,

$$
E(n)=2(2 n r+1)^{d} z(N, \delta)+e^{-\varepsilon N \delta} \sum_{k \geqq n} \frac{(M N \delta)^{k}}{k !}
$$

and

$$
E(N, \delta)=\min _{n \geqq 1} E(n)
$$

The minimizing $n$ in (3.4) is a characteristic length for the IPS: for any site $i \in \mathbb{Z}^{d}$, if its distance to the origin (in units of the interaction radius $r$ ) exceeds this length, then its influence on the behavior of the IPS at the origin at time $N \delta$ is, at most, of the order of $z(N, \delta)$. This will become clear in Sect. 7. Just note here that $n \geqq M N \delta$,

$$
E(n) \leqq 2(2 n r+1)^{d} z(N, \delta)+\frac{(M N \delta)^{n}}{n !} e^{-\varepsilon N \delta}\left[1-\frac{M N \delta}{n+1}\right]^{-1}
$$

so that in case $\delta \ll 1 \ll N \delta$, using $n \approx M N \delta$ in the right-hand side of (3.5) gives a good and explicit upper bound for (3.4).

As in (2.26) but for the $\delta$ PCA, let $Q_{\delta}\left[\eta_{N}(i) \neq\left(\eta^{j}\right)_{N}(i)\right]$ denote the probability with respect to the coupling $\delta$ PCA that at time $N$ the spin values at site $i \in \mathbb{Z}^{d}$ are different. Here, $\eta_{0}=\eta,\left(\eta^{j}\right)_{0}=\eta^{j}, \eta \in \Omega, j \in \mathbb{Z}^{d}$. Put

$$
k_{i j}^{(\delta) N}=\sup _{\eta} Q_{\delta}\left[\eta_{N}(i) \neq\left(\eta^{j}\right)_{N}(i)\right]
$$

and

$$
k_{j}^{(\delta) N}=k_{o j}^{(\delta) N}
$$

We let

$$
\gamma_{N}^{(\delta)}=\sum_{j} k_{j}^{(\delta) N}
$$

Definition. We say that condition $C_{N}^{\delta}$ holds for the IPS (2.16)-(2.19) if

$$
\gamma_{N}^{(\delta)}<1-E(N, \delta)
$$

Theorem A. Suppose that for the IPS there exist $\delta$ and $N$ as above, such that condition $C_{N}^{\delta}$ holds. Then (not only the $\delta$ PCA but also) the IPS is ergodic, and for some $c, \lambda>0$, for all $f \in D(\Omega), t>0$,

$$
\left\|S(t) f-\int v(d \eta) f(\eta)\right\| \leqq c e^{-i t}\|\| f\|\|,
$$

where $v$ is the unique invariant measure.

In the rest of the paper, IPS for which (3.10) is satisfied for all local functions $f$, will be called uniformly exponentially ergodic (UEE).

Remarks.

1. From Theorem $\mathrm{C}$ in the next section it follows immediately that the approximating PCA process of Theorem A - which is the analog of the so-called Euler polygonal approximation from ordinary differential equations - is ergodic. For this 
we only use that $\gamma_{N}^{(\delta)}<1$. We need the gap $1-\gamma_{N}^{(\delta)}$ to be large enough to get that in that case the approximated process (the IPS) is also ergodic.

2. We will show in Sect. 7 that the limit $\delta \downarrow 0$ of condition $C_{1}^{\delta}(N=1)$ yields the well known $M<\varepsilon$ condition [L]. Next hope would be that the limit $\delta \downarrow 0$ of $C_{N}^{\delta}$ for $N>1$ give new (and better) ergodicity criteria. This however is not true: by this procedure one cannot go beyond the $M<\varepsilon$ condition. In general we have that $\gamma_{N}^{(\delta)}=1+N(M-\varepsilon) \delta+O\left(\delta^{2}\right)$. One only arrives at improved ergodicity criteria by keeping $\delta>0$ fixed, though small. This is possible because $z(N, \delta)$ starts off quadratically in $\delta$ for fixed $N$, see (6.27). In fact, as we will show below, for attractive IPS also the opposite statement holds true: if the IPS is UEE then, for some $\delta, N$, the condition $C_{N}^{\delta}$ is verified.

Theorem B. Suppose that for the IPS (2.16)-(2.19), its Vasserstein coupling $T$ of Sect. 2d), satisfies

$$
\lim _{t \uparrow \infty} t^{d} \sup _{\eta, j} T^{\eta, \eta^{j}}\left[\eta_{t}(o) \neq\left(\eta^{j}\right)_{t}(o)\right]=0 .
$$

Then, for some $\delta>0, N=1,2, \ldots$ the IPS satisfies condition $C_{N}^{\delta}$ and hence, this IPS is UEE.

Actually, we can prove slightly more. Choose a constant $\tau(M)$ such that for all $t \geqq \tau(M)$,

$$
\frac{(M T)^{3 M t}}{[3 M t] !}<\frac{1}{10} .
$$

Then the statement of Theorem B remains true if we suppose only that for some $t \geqq \tau(M)$,

$$
(6 M r t+1)^{d} \sup _{\eta \cdot j} T^{\eta, \eta^{j}}\left[\eta_{t}(o) \neq\left(\eta^{j}\right)_{t}(o)\right]<\frac{1}{3} .
$$

A similar remark holds for the next corollary which is easy to prove from (2.27).

Corollary 1. Suppose that for an attractive IPS, as $t \uparrow \infty$,

$$
S(t)(+)-S(t)(-)=o\left(t^{-d}\right) .
$$

Then, this IPS satisfies condition $C_{N}^{\delta}$ for some $\delta>0$ and $N$ and hence, is UEE.

This last result is also contained in $[\mathrm{AH}]$ and $[\mathrm{H}]$. Proofs of Theorems A and $\mathrm{B}$ will be given in Sect. 7 .

\section{Ergodicity of PCA}

Let a PCA, defined by (2.7)--(2.11) be given, and choose an integer $N>0$. Put

and

$$
k_{i j}^{N}=\sup _{\eta} Q^{\eta, \eta^{\prime}}\left[\sigma_{N}(i) \neq \bar{\sigma}_{N}(i)\right]
$$

$$
k_{j}^{N}=k_{o j}^{N} .
$$


Theorem C. Suppose that condition $C_{N}$ holds:

$$
\gamma_{N}=\sum_{j} k_{j}^{N}<1
$$

Then the PCA is ergodic and for all $f \in D(\Omega), 0<a, 0 \leqq b<N$,

$$
\left\|P^{a N+b} f-\int f(\eta) v(d \eta)\right\| \leqq \frac{\left(\gamma_{N}\right)^{a}}{1-\gamma_{N}}\left\|P^{b} f\right\| \|,
$$

where $v$ is the unique invariant measure.

Remarks.

1. An advantage of Theorem $\mathrm{C}$ over results of $[\mathrm{MS}]$ is that the condition $C_{N}$ of (4.3) is much simpler than the conditions $C_{N, R}$ introduced there.

2. Theorem $\mathrm{C}$ is easy to extend to the non-translation invariant case with non-finite interaction radius. In that case

$$
\gamma_{N}=\sup _{i} \sum_{j} k_{i j}^{N}
$$

3. The case $N=1$ is the usual Vasserstein-Dobrushin single site condition $([\mathrm{V}, \mathrm{D}, \mathrm{LMS}])$ since it is readily checked that

$$
k_{i j}^{1}=\sup _{\eta}\left|p_{i}\left(\eta^{j}\right)-p_{i}(\eta)\right| .
$$

Then,

$$
\gamma_{1}=\sum_{j} \sup _{\eta}\left|p_{o}\left(\eta^{j}\right)-p_{o}(\eta)\right|
$$

4. Notice that condition $C_{N}$ can be viewed as a property of an auxiliary PCA defined on a large (in fact, twice as large) state space. It is sufficient to work with just one coupling PCA and to make a finite time calculation in order to check whether (4.3) holds. We can thus say that Theorem $\mathrm{C}$ relates the asymptotic behavior of a PCA to the finite time $(=N)$ behavior of a coupling PCA.

5. For attractive PCA, cf. (2.27) and the remark below,

where

$$
k_{i j}^{N}=\sup _{\eta}\left|P_{i}^{N}\left(\eta^{j}\right)-P_{i}^{N}(\eta)\right|,
$$

$$
P_{i}^{N}(\eta)=\operatorname{Prob}\left[\sigma_{N}(i)=1 \mid \sigma_{0}=\eta\right]
$$

is the probability that $\sigma_{N}(i)=1$ when started at time zero from the configuration $\sigma_{0}=\eta$. In particular, $k_{i j}^{N}=0$ unless $j \in W(i, N)$, the cube in $\mathbb{Z}^{d}$ around site $i$ with sidelength $2 N r+1$. If $j \in W(i, N)$ we can use

$$
k_{i j}^{N} \leqq \sup _{j, \eta}\left|P_{i}^{N}\left(\eta^{j}\right)-P_{i}^{N}(\eta)\right| \leqq P_{i}^{N}(+)-P_{i}^{N}(-),
$$

where, as in (2.27), the arguments \pm denote the all plus, respectively, all minus configurations. It is then easy to prove

Corollary 2. For an attractive PCA, suppose that there exists an integer $\eta>0$ such that

$$
P^{n}(+)-P^{n}(-)<\frac{1}{(2 n r+1)^{d}} .
$$

Then, the PCA satisfies condition $C_{n}$ and hence, is UEE (in the sense of (4.3)). 
Remark. Usually this kind of statement is formulated with $o\left(n^{-d}\right)$ in the right-hand side of (4.10), see e.g. (3.12). Our slight improvement here is that it is actually enough for exponentially fast decay to have a $c n^{-d}$-decay with $c$ sufficiently small.

Proof of Theorem C. By standard arguments (see the proof of Theorem 1 in [LMS]) it is sufficient to show the contraction property $\left|\left\|P^{N} f\right\|\right| \leqq \gamma_{N}|\|f \mid\|$. But, using the coupling PCA defined in Sect. 2d) on the strip $S_{N}$,

$$
P^{N} f\left(\eta^{j}\right)-P^{N} f(\eta)=\int\left[f\left(\bar{\sigma}_{N}\right)-f\left(\sigma_{N}\right)\right] \rho_{N}\left(d \sigma, d \bar{\sigma} \mid \eta, \eta^{j}\right)
$$

so that

$$
\left|P^{N} f\left(\eta^{j}\right)-P^{N} f(\eta)\right|=\int \sum_{i \in \mathbb{Z}^{d}: \sigma_{N}(l) \neq \bar{\sigma}_{N}(l)} \delta_{i} f \rho_{N}\left(d \sigma, d \bar{\sigma} \mid \eta, \eta^{j}\right)
$$

and

$$
\delta_{j} P^{N} f \leqq \sum_{i} \delta_{i} f k_{i j}^{N}
$$

which after summing over $j \in \mathbb{Z}^{d}$ gives the desired result.

Examples. We illustrate Theorem $\mathrm{C}$ by applying the criterion $\gamma_{N}<1$ to three specific models. All three are parametrized by a constant $0 \leqq \lambda \leqq 1$ and, as will be clear, each time Eq. (4.7) can be used.

1. Stavskaya's model is a one-dimensional PCA with

$$
\begin{aligned}
p_{o}(\eta) & =1 & & \text { if } \eta(-1)=\eta(0)=1 \\
& =\lambda & & \text { otherwise } .
\end{aligned}
$$

For all the cases considered $(N=1,2,3,4)$ we find that $k_{j}^{N}=P^{N}(+)-P^{N}\left(+^{j}\right)=$ $1-P^{N}\left(+{ }^{j}\right)$ and

$$
\begin{aligned}
& \gamma_{1}=2(1-\lambda) \\
& \gamma_{2}=(1-\lambda)^{2}(3+\lambda), \\
& \gamma_{3}=4(1-\lambda)^{3}(1+\lambda), \\
& \gamma_{4}=(1-\lambda)^{4}\left(5+9 \lambda+4 \lambda^{2}-3 \lambda^{3}+\lambda^{4}\right) .
\end{aligned}
$$

It implies ergodicity for that model (from $\gamma_{1}$ ) for $\lambda>0.5$ and (from $\gamma_{4}$ ) for $\lambda \geqq 0.45$ $\left(\gamma_{4}(\lambda=0.45)=0.95\right)$. The $[\mathrm{MS}]$ estimate gives ergodicity for $\lambda \geqq 0.484$.

2. The Majority Vote Model in one dimension is the PCA with

$$
\begin{aligned}
p_{o}(\eta) & =\frac{1+\lambda}{2} \text { if } \eta(-1)+\eta(0)+\eta(-1)>0 \\
& =\frac{1-\lambda}{2} \text { otherwise } .
\end{aligned}
$$

Here are

$$
\begin{aligned}
& \gamma_{1}=3 \hat{\lambda}, \\
& \gamma_{2}=3 \lambda^{2}-\lambda^{4},
\end{aligned}
$$


and the supremum in (4.7) is reached for $\eta$ 's which are \pm alternating. Equation (4.17) implies ergodicity for, respectively, $\lambda \leqq 0.33$ and $\lambda \leqq 0.61$. [MS] gives ergodicity for $\lambda \leqq 0.366$.

3. Toom's model is a two-dimensional north-east-center majority vote model. That is the PCA with

$$
\begin{aligned}
p_{o}(\eta) & =\frac{1+\lambda}{2} \text { if } \eta(1,0)+\eta(0,0)+\eta(0,1)>0 \\
& =\frac{1-\lambda}{2} \text { otherwise. }
\end{aligned}
$$

The calculations are quite similar to the previous model but here

$$
\begin{aligned}
& \gamma_{1}=3 \lambda, \\
& \gamma_{2}=5 \lambda^{2}+i^{4}
\end{aligned}
$$

implying ergodicity for, respectively, $\lambda \leqq 0.33$ and $\lambda \leqq 0.43$. [MS] gives the same result for $\lambda \leqq 0.352$. In all three examples the bounds of [MS] have been improved.

\section{The Locality of the Process}

We have assumed that both the discrete time and continuous time processes have a finite range of interaction $r$, see (2.7), (2.8) and (2.17). Clearly however, there is a difference in the dependence of the time evolved configuration on the initial data.

For PCA, this dependence is strictly local. The spin $\sigma_{n}(o)$ at the origin is determined at time $n \geqq 0$ by the values $\sigma_{0}(j), j \in([-n r, n r] \cap \mathbb{Z})^{d}$, of the spins at time zero in a finite set only. In other words, for all $n \geqq 0, P^{n} f$ is local whenever $f$ is a local function.

For the continuous time versions, if $f \in D(\Omega)$, then, for all $t \geqq 0, S(t) f \in D(\Omega)$, see [L], but locality of the function is not preserved. Since we have to deal with this problem in later sections, we summarize here how much $S(t) f(\eta)$ can depend on $\eta(j)$ for far away $j \in \mathbb{Z}^{d}$.

This information can be extracted from Theorem I.3.9(c) in [L] giving

$$
\delta_{j} S(t) f \leqq e^{-\varepsilon t} \sum_{i \in \mathbb{Z}^{d}} \exp [t \Gamma](i, j) \delta_{i} f,
$$

where $\Gamma$ is the bounded operator on $l_{1}\left(\mathbb{Z}^{d}\right)$ with norm $M$, defined via its matrix elements

$$
\begin{aligned}
\Gamma(i, j) & =\sup _{\eta}\left|c\left(i, \eta^{j}\right)-c(i, \eta)\right| & & \text { if } i \neq j, \\
& =0 & & \text { if } i=j .
\end{aligned}
$$

Using the idea of coupling it is easy to see why (5.1) holds true. In showing this below, we at the same time will obtain the (slightly stronger) information which we will use in Sect. 7.

Let $T^{\eta, \bar{\eta}}$ denote the Vasserstein coupling (see III.1 in [L] or Sect. 2d) above) between the processes $\eta_{t}$ and $\bar{\eta}_{t}$ both having spinflip rates $(c(i, \eta)$, but starting from configurations $\eta$, respectively, $\bar{\eta}$. 
For notational convenience we will write

$$
\begin{aligned}
& T^{\eta, \bar{\eta}}\left[f\left(\eta_{t}, \bar{\eta}_{t}\right) ; \eta_{t}(i)= \pm \bar{\eta}_{t}(i)\right] \\
& =\int f\left(\eta_{t}, \bar{\eta}_{t}\right) T^{\eta, \bar{\eta}}\left[d \eta_{t}, d \bar{\eta}_{t} \mid \eta_{t}(i)= \pm \bar{\eta}_{t}(i)\right] T^{\eta, \bar{\eta}}\left[\eta_{t}(i)= \pm \bar{\eta}_{t}(i)\right] .
\end{aligned}
$$

Then,

$$
\begin{aligned}
& \frac{d}{d t} T^{\eta, \bar{\eta}}\left[\eta_{t}(i) \neq \bar{\eta}_{t}(i)\right] \\
& =T^{\eta, \bar{\eta}}\left[\left|c\left(i, \eta_{t}\right)-c\left(i, \bar{\eta}_{t}\right)\right| ; \eta_{t}(i)=\bar{\eta}_{t}(i)\right]-T^{\eta, \bar{\eta}}\left[c\left(i, \eta_{t}\right)+c\left(i, \bar{\eta}_{t}\right) ; \eta_{t}(i) \neq \bar{\eta}_{t}(i)\right] \\
& \leqq \sum_{k} \Gamma(i, k) T^{\eta, \bar{\eta}}\left[\eta_{t}(k) \neq \bar{\eta}_{t}(k)\right]-\varepsilon T^{\eta, \bar{\eta}}\left[\eta_{t}(i) \neq \bar{\eta}_{t}(i)\right] .
\end{aligned}
$$

For the inequality we have used that for local functions $f, \sigma, \bar{\sigma} \in \Omega$

$$
|f(\sigma)-f(\bar{\sigma})| \leqq \sum_{k: \sigma(k) \neq \bar{\sigma}(k)} \delta_{k} f
$$

together with definitions (5.2) and (2.18). Hence,

$$
\frac{d}{d t}\left[e^{\varepsilon t} \sum_{k} e^{-\Gamma t}(i, k) T^{\eta, \bar{\eta}}\left[\eta_{t}(k) \neq \bar{\eta}_{t}(k)\right]\right] \leqq 0
$$

or, taking $\bar{\eta}=\eta^{j}$,

$$
T^{\eta, \eta^{j}}\left[\eta_{t}(i) \neq\left(\eta^{j}\right)_{t}(i)\right] \leqq e^{-\varepsilon t} e^{\Gamma t}(i, j) .
$$

It is clear that (5.1) follows from this.

A similar derivation shows that for the discrete time case

$$
Q^{\eta, \eta^{j}}\left[\eta_{n}(i) \neq\left(\eta^{j}\right)_{n}(i)\right] \leqq\left(\delta_{o} p_{o}+K\right)^{n}(i, j),
$$

where $K$ has matrix elements $K(i, j)=\delta_{j} p_{i}$ if $i \neq j$ and zero otherwise.

\section{Approximating Continuous Time Spinflip Processes by PCA}

Given the spinflip process (2.16)-(2.19) with rates $c(i, \eta)$, we define a family of approximating PCA $\eta_{n}^{(\delta)}$ indexed by $\delta>0$. They are the $\delta$ PCA of Sect. 3. Each of those has spinflip probabilities

$$
\operatorname{Prob}\left[\eta_{n+1}^{(\delta)}(i) \neq \eta_{n}^{(\delta)}(i) \mid \eta_{n}^{(\delta)}=\eta\right]=\frac{1}{2}(1-\exp [-2 \delta c(i, \eta)])
$$

In other words, the transition probabilities (2.9) are equal to (3.1) and of the form

$$
\begin{aligned}
p_{i}^{(\delta)}(\eta) & =\delta c(i, \eta)+o(\delta) & & \text { if } \eta(i)=-1, \\
& =1-\delta c(i, \eta)+o(\delta) & & \text { if } \eta(i)=1 .
\end{aligned}
$$

Obviously, many other choices than (6.1) can be made giving rise to (6.2), the simplest of which would be to let the spinflip probabilities equal $\delta c(i, \eta)$. Our choice has the advantage of being more natural for the lemmas that follow and giving better estimates. 
The associated transition operator (2.11) is denoted by $P_{\delta}$, e.g. if $f(\sigma)=\sigma(o)$, then, ignoring higher orders in $\delta$,

$$
\begin{aligned}
P_{\delta} f(\eta) & \approx[\delta c(o, \eta)(1-\eta(o))+(1-\delta c(o, \eta))(1+\eta(o))]-1 \\
& =\eta(o)-2 \delta c(o, \eta) \eta(o) \\
& =[I+\delta L] f(\eta),
\end{aligned}
$$

where $I$ stands for the identity operator.

It is easy to check that (6.3) remains valid for any local function $f$ up to first order in $\delta$. Further details and a proof of the following lemma can be found in [St] as a direct application of this fact:

Lemma 1. As $\delta \downarrow 0$,

a) For all $f \in D(\Omega)$,

$$
\frac{1}{\delta}\left[P_{\delta}-I\right] f \rightarrow L f
$$

b) For all $f \in C(\Omega)$,

$$
P_{\delta}^{[t / \delta]} f \rightarrow S(t) f
$$

for all $t \geqq 0$ uniformly on compacts; $[t / \delta]$ is the smallest integer greater than or equal to $t / \delta$.

In the following sections we need some more details on just what the correction is to (6.5) for finite $\delta>0$. This will be done in Corollary 3 below.

For that purpose we introduce an auxiliary continuous time spinflip process $\Xi_{t}^{\sigma}, 0 \leqq t \leqq \delta$, depending on a fixed configuration $\sigma \in \Omega$ in two ways. First, we require the process to start from it, $\Xi_{0}^{\sigma}=\sigma$, and, secondly, the spinflip rates (denoted by $s(i, \Xi)$ ) for that process are constant (in the second argument $\Xi$ ) equal to

$$
s(i, \Xi)=c(i, \sigma) .
$$

It is easy to verify from (6.6) that the measure at time $t$ is a product measure with

$$
\operatorname{Prob}\left[\Xi_{t}^{\sigma}(i) \neq \sigma(i)\right]=\frac{1}{2}(1-\exp [-2 t c(i, \sigma)]), \quad 0 \leqq t \leqq \delta
$$

which imitates formula (6.1).

On this same time interval we also consider the original process $\eta_{t}$ with spinflip rates $c(i, \eta)$, started from some configuration $\eta_{0}=\eta \in \Omega$.

Let $A^{\eta, \sigma}$ denote the Vasserstein coupling between these two processes $\eta_{t}$ and $\Xi_{t}^{\sigma}$, see e.g. III.1 in [L]. We choose a probability measure $m(d \eta, d \sigma)$ on $\Omega \times \Omega$ as initial measure for the coupled process $A^{m}=\int m(d \eta, d \sigma) A^{\eta, \sigma}$ and define for $t \in[0, \delta]$

$$
e_{t}=\sup _{i \in \mathbb{Z}^{d}} A^{m}\left[\eta_{t}(i) \neq \Xi_{t}^{\sigma}(i)\right]
$$

\section{Lemma 2.}

$$
e_{\delta} \leqq e^{M \delta} e_{0}+\frac{B}{M}\left(e^{M \delta}-1\right)-\frac{1}{2}\left(1-e^{-2 B \delta}\right) .
$$

Note that the right-hand side of (6.9) is $O\left(\delta^{2}\right)$ if $e_{0}=0$. 
Proof of Lemma 2. We proceed as in (5.3) and use the same notation as explained above (5.3). For any $h>0$,

$$
\begin{aligned}
A^{\eta \cdot \sigma}\left[\eta_{t+h}(i) \neq\right. & \left.\Xi_{t+h}^{\sigma}(i)\right]=A^{\eta \cdot \sigma}\left[\eta_{t+h}(i) \neq \Xi_{t+h}^{\sigma}(i) ; \eta_{t}(i)=\Xi_{t}^{\sigma}(i)\right] \\
& +A^{\eta, \sigma}\left[\Xi_{t+h}^{\sigma}(i) \eta_{t+h}(i) \neq \Xi_{t+h}^{\sigma}(i) \mid \eta_{t}(i) \neq \Xi_{t}^{\sigma}(i)\right] A^{\eta, \sigma}\left[\eta_{t}(i) \neq \Xi_{t}^{\sigma}(i)\right] \\
\leqq & h A^{\eta, \sigma}\left[\left|c(i, \sigma)-c\left(i, \eta_{t}\right)\right| ; \eta_{t}(i)=\Xi_{t}^{\sigma}(i)\right]+o(h)+A^{\eta, \sigma}\left[\eta_{t}(i) \neq \Xi_{t}^{\sigma}(i)\right]
\end{aligned}
$$

as follows from the definition of Vasserstein coupling together with (7.6). Moreover, via the triangle inequality,

$$
\begin{aligned}
A^{\eta, \sigma}\left[\left|c(i, \sigma)-c\left(i, \eta_{t}\right)\right| ; \eta_{t}(i)=\Xi_{t}^{\sigma}(i)\right] \leqq & A^{\eta, \sigma}\left[\left|c(i, \sigma)-c\left(i, \Xi_{t}^{\sigma}\right)\right| ; \eta_{t}(i)=\Xi_{t}^{\sigma}(i)\right] \\
& \left.+A^{\eta, \sigma}\left[\left|c\left(i, \Xi_{t}^{\sigma}\right)-c\left(i, \eta_{t}\right)\right| ; \eta_{t}(i)=\Xi_{t}^{\sigma} i\right)\right] .
\end{aligned}
$$

On the other hand, we can apply inequality (5.4) to (6.11) and combine it with the definitions $\delta_{j} c(i, \cdot)=\Gamma(i, j), i \neq j, \Gamma(i, i)=0$ to get

$$
\begin{aligned}
(6.11) \leqq & \sum_{j} \Gamma(i, j) A^{\eta, \sigma}\left[\sigma(j) \neq \Xi_{t}^{\sigma}(j)\right]+\delta_{i} c(i, \cdot) A^{\eta, \sigma}\left[\sigma(i) \neq \Xi_{t}^{\sigma}(i)\right] \\
& +\sum_{j} \Gamma(i, j) A^{\eta, \sigma}\left[\eta_{t}(j) \neq \Xi_{t}^{\sigma}(j)\right] .
\end{aligned}
$$

Of course, in the first two terms the expectation only involves the second marginal and moreover, we know from the definition of the $\Xi_{t}^{\sigma}$ process and (6.7) that for all $j \in \mathbb{Z}^{d}$

$$
A^{\eta, \sigma}\left[\sigma(j) \neq \Xi_{t}^{\sigma}(j)\right]=\frac{1}{2}(1-\exp [-2 t c(j, \sigma)]) \leqq \frac{1}{2}\left(1-e^{-2 B t}\right) .
$$

Combining (6.10)-(6.13) we get, upon averaging with $m(d \eta, d \sigma)$,

$$
e_{t+h} \leqq h(M+2 B) \frac{1}{2}\left(1-e^{-2 B t}\right)+h M e_{t}+o(h)+e_{t} .
$$

Hence, taking $h \downarrow 0$,

$$
\frac{d}{d t}\left(e^{-M t} e_{t}\right) \leqq\left(\frac{M}{2}+B\right) e^{-M t}\left(1-e^{-2 B t}\right)
$$

which after integration over $0 \leqq t \leqq \delta$ gives the desired result.

There clearly is a connection between the process $\Xi_{t}^{\sigma}$ just considered and the approximating PCA $\eta_{n}^{(\delta)}$, cf. (6.7). To construct a stochastic process $\sigma_{t}$ for which, at all times $t=n \delta$,

$$
\sigma_{t}=\eta_{n}^{(\delta)}
$$

in distribution, we must repeat the above construction in all intervals of the form $[n \delta,(n+1) \delta]$. This means that we take $\sigma_{t}$ to be the right continuous process (which is no longer Markovian) having, in the interval $[n \delta,(n+1) \delta]$, spinflip rates $c\left(i, \sigma_{n \delta}\right)$, or,

$$
\sigma_{t}=\Xi_{s}^{\sigma_{n \delta}}, \quad t=n \delta+s, \quad 0 \leqq s \leqq \delta
$$


This construction allows us to prove the following

Lemma 3. For all $f \in D(\Omega)$,

$$
\left\|S(N \delta) f-P_{\delta}^{N} f\right\| \leqq z(N, \delta)\|\| f\|\|,
$$

where

$$
z(N, \delta)=\frac{B}{M}\left(e^{M N \delta}-1\right)-\frac{1}{2} \frac{1-e^{-2 B \delta}}{e^{M \delta}-1}\left(e^{M N \delta}-1\right) .
$$

Proof of Lemma 3. In analogy with the coupling we had in Lemma 2 between $\eta_{t}$ and $\Xi_{t}^{\sigma}$, we let $\bar{A}^{\eta}$ denote the modification to a coupling between $\eta_{t}$ and $\sigma_{t}$, both started from configuration $\eta$. This modification (also non-Markovian) is easy to realize by applying the same idea as in (6.16)-(6.17). Define

$$
v_{n}=\sup _{i} \bar{A}^{\eta}\left[\eta_{n \delta}(i) \neq \sigma_{n \delta}(i)\right]
$$

so that $v_{0}=0$ and, from (6.9),

$$
v_{n} \leqq e^{M \delta} v_{n-1}+\frac{B}{M}\left(e^{M \delta}-1\right)-\frac{1}{2}\left(1-e^{-2 B \delta}\right) .
$$

Hence,

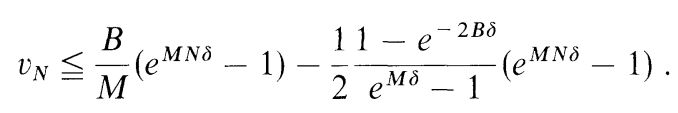

This finishes the proof because

$$
\left|S(t) f(\eta)-P_{\delta}^{N} f(\eta)\right| \leqq \sum_{i} \delta_{i} f \bar{A}^{\eta}\left[\eta_{t}(i) \neq \sigma_{t}(i)\right]
$$

and, for $t=N \delta$, (6.23) is bounded by $v_{N}\|\mid f\| \|$.

The general estimate controlling (6.5) for times $t$ which are not exact multiples of $\delta$ is given in the following

Corollary 3. For all values of $t, \delta>0$ and for all $f \in D(\Omega)$,

$$
\left\|S(t) f-P_{\delta}^{[t / \delta]} f\right\| \leqq[z([t / \delta], \delta)+\delta B]\|f\| \| .
$$

Proof of Corollary 3. Suppose that $t=N \delta-s$, where $0 \leqq s<\delta$ so that $N=[t / \delta]$. Then,

$$
\begin{aligned}
\left\|S(t) f-P_{\delta}^{[t / \delta]} f\right\| & \leqq\|S(N \delta) f-S(t) f\|+\left\|S(N \delta) f-P_{\delta}^{N} f\right\| \\
& \leqq\|S(s) f-I f\|+z(N, \delta)\|f\| \| .
\end{aligned}
$$

Finally,

$$
\|S(s) f-I f\| \leqq s\|L f\| \leqq \delta B\|f\| \|
$$

Remark. 1. It is important that for fixed $t=N \delta$,

$$
z(N, \delta)=\left(e^{M t}-1\right) \frac{B}{M}\left[\left(\frac{M}{2}+B\right) \delta+O\left(\delta^{2}\right)\right]
$$

can be made arbitrarily small by making $\delta>0$ sufficiently small. 
2. A slight modification of the above arguments (starting with a better estimate in (6.10)) shows that (6.18) and (6.24) remain true for

$$
\begin{aligned}
z(N, \delta)= & \frac{(M+2 B) B}{(M-\varepsilon)(M-\varepsilon+2 B)}\left(e^{(M-\varepsilon) N \delta}-1\right) \\
& -\frac{M / 2+B}{M-\varepsilon+2 B} \frac{1-e^{-2 B \delta}}{e^{(M-\varepsilon) \delta}-1}\left[e^{(M-\varepsilon) N \delta}-1\right]
\end{aligned}
$$

replacing definition (6.19). Notice from $(6.28)$ that $z([t / \delta], \delta)$ is bounded uniformly in $t$ whenever $M<\varepsilon$. More generally, we expect that the PCA discretization is $O(\delta)$ close to the original continuous time process, uniformly in time, if this last process is ergodic.

\section{When is a Spinflip Process Ergodic?}

The best known ergodicity criterion for interacting particles is the so-called " $M<\varepsilon$ "-condition (see Theorem I 4.1 in [L]). $M$ and $\varepsilon$ are the quantities defined in (2.18). The traditional proof (as in [L]) of this condition is rather involved even in the case of spinflip processes. Therefore we start our investigation by rederiving the " $M<\varepsilon$ "-condition with a minimal amount of work using [St]'s idea of approximating with PCA.

From Theorem $\mathrm{C}$ and (4.5) we know that the approximating PCA is ergodic if

$$
\gamma_{1}^{(\delta)}=\sum_{j} \sup _{\eta}\left|p_{o}^{(\delta)}\left(\eta^{j}\right)-p_{o}^{(\delta)}(\eta)\right|<1 .
$$

Easy calculations show that (6.2) implies that

$$
\begin{aligned}
& \sum_{j \neq 0} \sup _{\eta}\left|p_{o}^{(\delta)}\left(\eta^{j}\right)-p_{o}^{(\delta)}(\eta)\right|=\delta M+o(\delta), \\
& \sup _{\eta}\left|p_{o}^{(\delta)}\left(\eta^{o}\right)-p_{o}^{(\delta)}(\eta)\right|=1-\delta \varepsilon+o(\delta),
\end{aligned}
$$

so that

$$
\gamma_{1}^{(\delta)}=1+\delta(M-\varepsilon)+o(\delta) .
$$

Hence, if $M<\varepsilon$, then $\gamma_{1}^{(\delta)}<1$ for $\delta$ small; moreover,

$$
\begin{aligned}
\|S(t) f\| \| & =\lim _{\delta \downarrow 0}\left\|P_{\delta}^{[t / \delta]} f\right\| \| \\
& \leqq \lim _{\delta \downarrow 0}\left[\gamma_{1}^{(\delta)}\right]^{[t / \delta]}\|f \mid\| \\
& =e^{t(M-\varepsilon)}|\|f \mid\|
\end{aligned}
$$

which is all what is needed in obtaining the " $M<\varepsilon$ "-condition.

One might be optimistic at this point and think that, for each $N$, a new condition for the ergodicity of continuous time processes is obtained from requiring that

$$
\lim _{\delta \downarrow 0}\left[\gamma_{N}^{(\delta)}\right]^{[t / N \delta]}<1
$$


Some thinking reveals however that this limit cannot give us anything better than for $N=1$. The reason is essentially that (7.5) requires the coefficient in front of $\delta$ in $\gamma_{N}^{(\delta)}$ to be negative but this coefficient only contains information about single spinflips no matter how large $N$ is, and is therefore governed (as for $N=1$ ) by the difference $M-\varepsilon$.

Example. Let a one-dimensional spinflip system be determined by the rates

$$
\begin{aligned}
c(i, \eta) & =0 & & \text { if } \eta(i-1)=\eta(i)=1, \\
& =M & & \text { if } \eta(i)=1, \quad \eta(i-1)=-1, \\
& =\varepsilon & & \text { if } \eta(i)=-1 .
\end{aligned}
$$

The parametrization of (7.6) is so arranged that $M$ and $\varepsilon$ have the same meaning as in (2.18). This model is an asymmetric contact process with infection rate $M / \varepsilon$, (Chapter VI in $[\mathrm{L}]$ ).

The $\delta$-approximating PCA has transition probabilities

$$
\begin{aligned}
p_{o}^{(\delta)}(\eta) & =1 & & \text { if } \eta(-1)=\eta(0)=1, \\
& =1-\alpha & & \text { if } \eta(0)=1, \quad \eta(-1)=-1, \\
& =\beta & & \text { if } \eta(0)=-1,
\end{aligned}
$$

where

$$
\alpha=\frac{1}{2}\left(1-e^{-2 \delta M}\right), \quad \beta=\frac{1}{2}\left(1-e^{-2 \delta \varepsilon}\right) .
$$

Using the methods of Sect. 4, we calculate

$$
\begin{aligned}
\gamma_{1}^{(\delta)}= & 1+\alpha-\beta \\
= & 1+(M-\varepsilon) \delta+\left(\varepsilon^{2}-M^{2}\right) \delta^{2}+O\left(\delta^{3}\right), \\
\gamma_{2}^{(\delta)}= & 1+2(\alpha-\beta)+\beta^{2}-2 \beta \alpha+\alpha^{2} \beta \\
= & \left.1+2(M-\varepsilon) \delta+\left(3 \varepsilon^{2}-2 M^{2}-2 \varepsilon M\right)\right) \delta^{2}+O\left(\delta^{3}\right), \\
\gamma_{3}^{(\delta)}= & 1+3(\alpha-\beta)+3 \beta^{2}-6 \alpha \beta+4 \alpha^{2} \beta+3 \alpha \beta^{2}-\beta^{3} \\
& -\alpha^{2} \beta^{2}+\alpha^{3} \beta^{2}+\alpha^{2} \beta^{3} \\
= & 1+3(M-\varepsilon) \delta+\left(3 \varepsilon^{2}-6 M \varepsilon\right) \delta^{2}+O\left(\delta^{3}\right) .
\end{aligned}
$$

We are obliged therefore to look in $\gamma_{N}^{(\delta)}$ for higher order than linear in $\delta$. To let these be effective, we should not take the limit $\delta \downarrow 0$ and we are thus facing the problem of estimating exactly (i.e. in constructive terms) how good the approximation is. This was solved in Sect. 6. Recall to that effect that $(3.2),(6.19)$ is $O\left(\delta^{2}\right)$ for fixed $N$.

In summary, our strategy consists in writing down the constructive criteria for ergodicity of an approximating PCA (as developed in Sect. 4) and then, to use the estimates of Sects. 5 and 6 to correct for the error made by the approximation.

Proof of Theorem $A$. From now on, we fix $\delta>0$ and the integer $N=1,2, \ldots$ We consider two initial configurations $\eta, \bar{\eta} \in \Omega$ and four continuous time processes, $\eta_{t}, \bar{\eta}_{t}, \sigma_{t}$ and $\bar{\sigma}_{t}$. The first two are the same spinflip processes (2.16)-(2.19) with rates $c(i, \cdot)$ but started from $\eta_{0}=\eta$ and $\bar{\eta}_{0}=\bar{\eta}$. Their PCA approximations are $\eta_{n}^{(\delta)}$, 
respectively $\bar{\eta}_{n}^{(\delta)}$, as defined in (6.1). Similarly, (6.16)-(6.17) define the processes $\sigma_{t}$ and $\bar{\sigma}_{t}$ with $\sigma_{0}=\eta$ and $\bar{\sigma}_{0}=\bar{\eta}$. We will always take $\bar{\eta}=\eta^{j}$ for some $j \in \mathbb{Z}^{d}$.

The spinflip processes $\eta_{t}, \bar{\eta}_{t}$ are easily coupled via the Vasserstein coupling $T^{\eta, \bar{\eta}}$ that we first considered in Sect. 2 d). For a fixed site $i \in \mathbb{Z}^{d}$ and integer $n>0$ consider the corresponding cube

$$
W=W(i, n)=\left\{j \in \mathbb{Z}^{d}:-n r \leqq j_{\alpha}-i_{\alpha} \leqq n r, \alpha=1, \ldots, d\right\},
$$

where $r$ is the radius of interaction defined in (2.8) and (2.17). The complement of $W$ in $\mathbb{Z}^{d}$ is denoted by $W^{c}$. We know from (5.6) that

$$
\begin{aligned}
\sum_{j \in W^{c}} T^{\eta, \eta^{j}}\left[\eta_{t}(i) \neq\left(\eta^{j}\right)_{t}(i)\right] & \leqq e^{-\varepsilon t} \sum_{k \geqq n} \frac{t^{k}}{k !} \sum_{j, J_{1}, \ldots, J_{k-1}} \Gamma\left(i, j_{1}\right) \ldots \Gamma\left(j_{k-1}, j\right) \\
& \leqq e^{-\varepsilon t} \sum_{k \geqq n} \frac{(M t)^{k}}{k !}
\end{aligned}
$$

The processes $\sigma_{t}$ and $\bar{\sigma}_{t}$ are not Markovian but we can still construct a coupling $R^{\eta, \tilde{\eta}}$ (also non-Markovian) between them. This is done by taking the coupling PCA $Q_{\delta}^{\eta, \bar{\eta}}$ of Sect. $2 \mathrm{~d}$ ) between $\eta_{n}^{(\delta)}$ and $\bar{\eta}_{n}^{(\delta)}$ and applying the same construction as in (6.16)-(6.17). For times $t=N \delta$,

$$
R^{\eta, \eta^{J}}\left[\sigma_{t}(i) \neq \bar{\sigma}_{t}(i)\right]=Q_{\delta}^{\eta, \eta^{j}}\left[\eta_{N}^{(\delta)}(i) \neq\left(\eta^{j}\right)_{N}^{(\delta)}(i)\right],
$$

and thus, as in (3.6)-(3.8),

$$
\sum_{j} R^{\eta, \eta^{j}}\left[\sigma_{t}(i) \neq \bar{\sigma}_{t}(i)\right] \leqq \gamma_{N}^{(\delta)}
$$

The four processes $\eta_{t}, \bar{\eta}_{t}, \sigma_{t}, \bar{\sigma}_{t}$ can be coupled together in a coupling $H^{\eta, \bar{\eta}}$ which is the modification of $A^{\eta, \bar{\eta}}$ of Lemmas 2 and 3 to a coupling between $T^{\eta, \bar{\eta}}$ and $R^{\eta \cdot \bar{\eta}}$.

Let $t=N \delta$. We are interested in

for $f \in D(\Omega)$.

$$
\delta_{j} S(t) f \leqq \sum_{i} \delta_{i} f \sup _{\eta} T^{\eta, \eta^{j}}\left[\eta_{t}(i) \neq \bar{\eta}_{t}(i)\right]
$$

The first case is when $j \notin W(i, n)$. Then we can use the bound (7.11).

The second case is when $j \in W(i, n)$. Then, we proceed via

$$
\begin{aligned}
T^{\eta, \eta^{\jmath}}\left[\eta_{t}(i) \neq \bar{\eta}_{t}(i)\right]= & H^{\eta, \eta^{\jmath}}\left[\eta_{t}(i)=\sigma_{t}(i) ; \bar{\eta}_{t}(i)=\bar{\sigma}_{t}(i) ; \sigma_{t}(i) \neq \bar{\sigma}_{t}(i)\right] \\
& +H^{\eta, \eta^{\jmath}}\left[\eta_{t}(i) \neq \sigma_{t}(i) \text { or } \bar{\eta}_{t}(i) \neq \bar{\sigma}_{t}(i) ; \eta_{t}(i) \neq \bar{\eta}_{t}(i)\right] \\
\leqq & R^{\eta, \eta^{j}}\left[\sigma_{t}(i) \neq \bar{\sigma}_{t}(i)\right]+2 \sup \bar{A}^{\eta}\left[\eta_{t}(i) \neq \sigma_{t}(i)\right]
\end{aligned}
$$

where, as before, it is understood that $\bar{\eta}=\eta^{j}$.

From the proof of Lemma 3, (6.22), we have

$$
\bar{A}^{\eta}\left[\eta_{t}(i) \neq \sigma_{t}(i)\right] \leqq z(N, \delta) .
$$

Combining (7.11)-(7.16) yields

$$
\||S(N \delta) f|\| \leqq\left[E(n)+\gamma_{N}^{(\delta)}\right]\|\mid f\| \| .
$$

Thus, minimizing (7.17) over $n$, we get that for all $\tau>0$,

$$
\||| S(\tau) f \mid\| \leqq \gamma^{\left[\frac{\tau}{N \delta}\right]-1} \sup _{0 \leqq s<N \delta}\|S(N \delta-s) f\| \|,
$$


where $\gamma=E(N, \delta)+\gamma_{N}^{(\delta)}<1$. Note that for $0 \leqq s<N \delta$,

$$
\|S(N \delta-s) f\|\left|\leqq e^{(M-\varepsilon)(N \delta-s)}\right|\|f\| \| \leqq \max \left\{1, e^{(M-\varepsilon) N \delta}\right\} .
$$

It is therefore easy to see that for any $0<t<T$,

$$
\begin{aligned}
\|S(T) f-S(t) f\| & \leqq \int_{t}^{T} d \tau\|L S(\tau) f\| \\
& \leqq \int_{t}^{T} d \tau B\|\mid S(\tau) f\| \| \\
& \leqq c e^{-i t} \mid\|f\| \|,
\end{aligned}
$$

where we define constants $0<\lambda \equiv-\frac{1}{N \delta} \ln \gamma, c \equiv \frac{B \max \left\{1, e^{(M-\varepsilon) N \delta}\right\}}{\lambda}<\infty$. The proof is concluded by noting that $\lim _{T} S(T) f$ exists (by (7.20)) and equals a constant (by (7.19)). The set $D(\Omega)$ is dense in $C(\Omega)$ and this constant must therefore equal the expectation value in the unique invariant measure.

Proof of Theorem $B$. We must show that $\gamma_{N}^{(\delta)}+E(N, \delta)<1$ for some $\delta, N$. From its definition

$$
\begin{aligned}
\gamma_{N}^{(\delta)} & =\sum_{j} \sup _{\eta} Q_{\delta}^{\eta, \eta^{j}}\left[\eta_{N}^{(\delta)}(o) \neq\left(\eta^{j}\right)_{N}^{(\delta)}(o)\right] \\
& \leqq \sum_{j \in W_{n}^{c}} \cdots+(2 n r+1)^{d} \sup _{\eta, j} R^{\eta, \eta^{j}}\left[\sigma_{t}(o) \neq \bar{\sigma}_{t}(o)\right]
\end{aligned}
$$

where we have cut the sum in two parts: in the first term $W_{n}^{c}$ is the complement of $W(o, n)$, see (7.10); for the last term, we have used (7.12) at time $t=N \delta$. We will choose the integer $n>0$ later.

From (5.7) and (7.2) for $\delta \ll 1$, a similar calculation as in (7.11) takes care of this first sum

$$
\begin{aligned}
\sum_{j \in W_{n}^{c}} Q_{\delta}^{\eta, \eta^{j}}\left[\eta_{N}^{(\delta)}(o) \neq\left(\eta^{j}\right)_{N}^{(\delta)}(o)\right] & \leqq\left[\delta_{o} p_{o}^{(\delta)}+K_{\delta}\right]^{N}(o, j) \\
& \leqq\left[1-\delta \varepsilon+O\left(\delta^{2}\right)\right]^{N} \sum_{j \in W_{n}^{c}}[1+\delta \Gamma]^{N}(o, j) \\
& \leqq\left[1-\delta \varepsilon+O\left(\delta^{2}\right)\right]^{N} \sum_{k \geqq n} \frac{(M N \delta)^{k}}{k !} .
\end{aligned}
$$

On the other hand, using the same notation as in the proof of Theorem A,

$$
\begin{aligned}
R^{\eta, \eta^{\jmath}}\left[\sigma_{t}(o) \neq \bar{\sigma}_{t}(o)\right] & \leqq T^{\eta, \eta^{\jmath}}\left[\eta_{t}(o) \neq\left(\eta^{j}\right)_{t}(o)\right]+2 \sup _{\eta} \bar{A}^{\eta}\left[\eta_{t}(o) \neq \sigma_{t}(o)\right] \\
& \leqq \gamma(t)+2 z(N, \delta)
\end{aligned}
$$

where, by hypothesis, $\gamma(t)=o\left(t^{-d}\right)$ as $t \uparrow \infty$. Summarizing the above, we have obtained that for all $n>0$,

$$
\begin{aligned}
\gamma_{N}^{(\delta)}+E(N, \delta) \leqq & {\left[1-\delta \varepsilon+O\left(\delta^{2}\right)\right]^{N} \sum_{k \geqq n} \frac{(M N \delta)^{k}}{k !}+(2 n r+1)^{d} \gamma(t) } \\
& +2(2 n r+1)^{d} z(N, \delta)+E(N, \delta) \\
\leqq & 2 E(n)+(2 n r+1)^{d} \gamma(t) .
\end{aligned}
$$


Now let $t=N \delta$ and choose $n=[3 M N \delta]=[3 M t]$. Take first $t$ very large so that the second term of (7.24) is less than $1 / 3$. We use (3.5) to estimate the remaining term. The second term in (3.5) is not greater than

$$
\frac{3(M t)^{3 M t}}{2[3 M t] !} e^{-\varepsilon t}
$$

which can be made less than $1 / 6$ by taking $t$ large. From (6.27), the first summand in (3.5) is bounded from above by

$$
2(6 M r t+1)\left(e^{M t}-1\right) \frac{B}{M}\left[\left(\frac{M}{2}+B\right) \delta+O\left(\delta^{2}\right)\right] .
$$

So it can be made less than $1 / 6$ by taking $\delta$ small enough. Hence the condition $C_{N}^{\delta}$ holds.

Acknowledgement. We thank the Institut de Physique Théorique, CNRS Luminy-Marseille where part of this work was done, for their kind hospitality.

\section{References}

[A] Aizenman, M.: Absence of an intermediate phase for a general class of one-component ferromagnetic models. Phys. Rev. Lett. 54, 839-842 (1985)

[ACCFR] Aizenman, M., Chayes, J.T., Chayes, L., Fröhlich, J., Russo, L.: On a sharp transition from area law to perimeter law in a system of random surfaces. Commun. Math. Phys. 92, 19-69 (1983)

[AH] Aizenman, M., Holley, R.: Rapid convergence to equilibrium of stochastic Ising models in the Dobrushin-Shlosman regime. In Percolation Theory and Ergodic Theory of Infinite Particle Systems, ed. H. Kesten; IMA Vol. Math. Appl. 8, Berlin, Heidelberg, New York: Springer 1987, pp. 1-11

[D] Dobrushin, R.L.: Markov processes with a large number of locally interacting components: Existence of a limit process and its ergodicity. Problems Inform. Transmission 7, 149-164 (1971)

[DS] Dobrushin, R.L., Shlosman, S.B.: Completely Analytic Interactions: Constructive Description. J. Stat. Phys. 46, 983-1014 (1987)

[H] Holley, R.: Possible rates of convergence in finite range, attractive spin systems. Contemp. Math. 41, 215-234 (1985)

[LMS] Lebowitz, J.L., Maes, C., Speer, E.R.: Statistical Mechanics of Probabilistic Cellular Automata. J. Stat. Phys. 59, 117-170 (1990)

[L] Liggett, T.M.: Interacting Particle Systems. Berlin, Heidelberg, New York: Springer 1985

[MS] Maes, C., Shlosman, S.B.: Ergodicity of Probabilistic Cellular Automata: A constructive criterion. Commun. Math. Phys. 135, 233-251 (1991)

[S] Spitzer, F.: Random processes defined through the interaction of an infinite particle system. Springer Lecture Notes in Mathematics 89, 201-223 (1969)

[St] Steif, J.: The Ergodic Structure of Interacting Particle Systems. Stanford University Ph.D. Thesis (1988)

[V] Vasserstein, L.N.: Markov Processes over Denumerable products of Spaces, Describing Large Systems of Automata. Problems Inform. Transmission 5, 47-52 (1969) 